\title{
Promoting diversity in neuroscience
}

\author{
Neuroscience is not spared from wrestling with gender disparity issues. Progress toward more balanced \\ representation has been slow, but improvement is possible with consistent and focused efforts.
}

n 2006, we published an editorial about the possible reasons for gender disparity in neuroscience. At that time, more than half of all neuroscience graduate trainees in the US were female, yet only $25 \%$ of tenure-track faculty in neuroscience were female and only one in five papers published in Nature Neuroscience had a female corresponding author ${ }^{1}$. In late 2016, after more than a decade of ongoing conversation about how to break down the barriers that might be limiting women's success in the laboratory, we reevaluated gender balance in our pool of authors and reviewers and developed a strategy for increasing diversity across several domains. Here, we report the results of our analyses and the improvements we have made since implementing our strategy.

We began by analyzing the primary research papers submitted to the journal from January through March of 2016. In that period, $21.5 \%$ of submitted manuscripts had a female corresponding author; this number is comparable to what we reported for the same three-month period in 2006 (20.5\%). Note that for the purpose of this analysis we inferred the corresponding author's likely gender based on their first name or through online searches, and we understand that our binary classification of gender does not reflect the full spectrum of gender identities. Both in 2006 and now, the chance that a paper from a female corresponding author was sent for peer review or ultimately accepted was the same as for a paper from a male corresponding author. We next assessed our referee pool, and we found that $18.5 \%$ of the reviewers in 2016 were female; this number represents only a modest increase from 2006, when the referee pool was $16.7 \%$ female. Finally, $17 \%$ (7 of 42 ) of our 2016 News \& Views were commissioned from female scientists, representing no improvement since 2006 when we also commissioned $17 \%$ (10 of 56) of the News \& Views from female scientists.

The 2016 Survey of Neuroscience Departments and Programs, conducted by the Society for Neuroscience, indicates that women still constitute more than half of all neuroscience graduate school trainees while representing 30\% of tenurestream faculty ${ }^{2}$. As of August 2015, https:// BiasWatchNeuro.com, a website dedicated to tracking minority representation in neuroscience, estimated the gender ratio of tenure-track faculty at large research universities at $24 \%$ female $^{3}$. Although gender disparity clearly persists in academia, our analyses indicate that we face an even larger disparity at the journal, including at the stage of submissions, but also in processes that we can control, such as reviewer panel enlistment and article commissioning.

Many of us have become familiar with the notion of implicit bias - the idea that underlying (and possibly unconscious) attitudes and associations can influence how we act. As editors, we are susceptible to implicit bias when we perform tasks such as inviting referees to papers and commissioning journal content. Implicit bias is pervasive, but it is possible to counteract it by acknowledging the problem and implementing proactive decision-making strategies. In late 2016, we resolved to make a concerted effort to increase female representation in our referee pool and in the authorship of News \& Views; we have continually reminded ourselves of this resolve throughout the last year. Now that 2017 has come to a close, we can report that these efforts have had a positive impact. In 2017, 28\% (13 of 46) of the News \& Views were commissioned from female scientists. Moreover, $34 \%$ of new referees (i.e., scientists who had not previously reviewed for Nature Neuroscience) were female. These numbers now roughly parallel the base rate of female faculty in neuroscience, and we will continually strive to improve this.

While our analysis suggests we have improved in the last year, the issue remains far from resolved. Specifically, we now need to expand these efforts beyond reviewer enlistment and News \& Views commissioning. Moreover, gender is only one facet of diversity. We are keenly aware of the need to consider minority status, geographical location, and seniority in diversifying our authorship and referee pool, and hope to tackle this more general problem now. We will continue to talk about implicit bias and work to counteract it, and we invite you to join us in our efforts. We have now added statements to our referee requests that explicitly remind scientists to consider diversity when recommending alternate referees. When we invite authors to write a Review or Perspective for our journal, we will urge them to assemble a diverse group of co-authors.

Beyond the boundaries of our journal, the problem of diversity persists in academia. We applaud the efforts of organizations like BiasWatchNeuro that are raising awareness of gender issues, and venues like the GRC Power Hour (https:// www.grc.org/the-power-hour/) that engage conference attendees in meaningful discussions about these pervasive issues. In fact, our own awareness of implicit bias was in part precipitated through our experiences in these forums. No one has an immediate solution to the problem, but awareness itself is the first step.

The conversation on diversity in neuroscience will continue. We call on the community, and ourselves, to strengthen actions to promote diversity in academia and in publishing. Our recent undertaking shows that change is possible when we put in a concerted effort. We welcome your feedback and thoughts on this important topic. You can write to us at neurosci@ us.nature.com.

Published online: 21 December 2017 https://doi.org/10.1038/s41593-017-0052-6

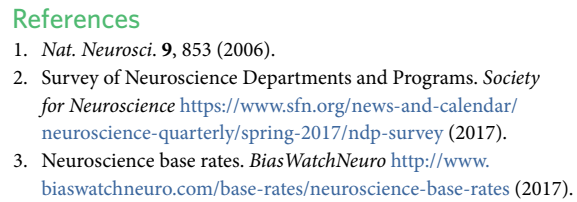

